\title{
Le protocole EMDR standard pour la dépendance à l'alcool et à d'autres substances psychoactives en comorbidité avec un état de stress post-traumatique : quatre cas avec un suivi après 12 mois
}

\author{
Claire Kullack \\ Jonathan Laugharne \\ École de psychiatrie et de neurosciences cliniques, université de l'Australie-Occidentale, Fremantle, \\ Australie-Occidentale, Australie
}

\begin{abstract}
Ce compte-rendu commence par un résumé de la littérature concernant les modèles théoriques qui soustendent la relation comorbide entre l'état de stress post-traumatique et les troubles de dépendance à des substances psychoactives, abordant également les divers protocoles modifiés pour les addictions, conçus pour aider au traitement de ces troubles. Cette série de cas donne un aperçu des effets que le protocole EMDR (désensibilisation et retraitement par les mouvements oculaires) standard a eu sur la dépendance à l'alcool et à d'autres substances psychoactives chez 4 patients de notre clinique du stress post-traumatique à Fremantle, en Australie-Occidentale, traités principalement pour un état de stress post-traumatique. Les patients ont été évalués pour des troubles addictifs à l'aide du Mini International Neuropsychiatric Interview Plus avant, immédiatement après et douze mois après la fin de la psychothérapie EMDR. Les résultats indiquent que le protocole EMDR standard a permis de réduire la consommation d'alcool et d'autres substances psychoactives. Avant le traitement, trois patients répondaient aux critères du syndrome de dépendance à l'alcool et un aux critères du syndrome de dépendance à une autre substance psychoactive. Lors du suivi après douze mois, trois patients sur quatre ne répondaient plus aux critères d'une dépendance présente à l'alcool ou d'une dépendance présente à une autre substance psychoactive. Les implications de ces résultats sont discutées au regard des théories de l'état de stress post-traumatique en comorbidité avec un syndrome de dépendance à des substances psychoactives et des protocoles EMDR modifiés développés pour les patients atteints d'une dépendance à des substances psychoactives.
\end{abstract}

Mots-clés : EMDR (désensibilisation et retraitement par les mouvements oculaires); protocole EMDR standard ; protocole EMDR modifié ; trouble de dépendance à une substance psychoactive ; état de stress post-traumatique

'état de stress post-traumatique (ESPT) est un trouble sévère, perturbant la vie de l'individu, qui peut se développer à la suite de l'exposition à un ou à plusieurs événements traumatiques. Il se caractérise par une constellation de symptômes de reviviscence, d'hyperactivation neurovégétative et d'évitement, ainsi que par des altérations négatives des cognitions et de l'humeur (American Psychiatric Association, 2013). L’ESPT peut avoir un effet délétère sur la qualité de vie et sur la capacité à entretenir des relations significatives et à maintenir un emploi (American Psychiatric Association, 2013).

Il est bien établi que les individus atteints d'ESPT montrent des taux accrus de troubles addictifs à des substances (Brady \& Sinha, 2005). Les taux de prévalence exacts rapportés varient considérablement, de $11 \%$ à $41 \%$ (Harrington \& Newman, 2007 ; Van

This article originally appeared as Kullack, C. \& Laugharne, J. (2016). Standard EMDR Protocol for Alcohol and Substance Dependence Comorbid With Posttraumatic Stress Disorder: Four Cases With 12-Month Follow-Up. Journal of EMDR Practice and Research, 10(1), 33-46. Translated by Jenny Ann Rydberg. 
Dam, Ehring, Vedel \& Emmelkamp, 2010). Kessler, Sonnega, Bromet, Hughes et Nelson (1995) ont montré que les hommes atteints d'un ESPT couraient deux fois plus de risques, par rapport aux hommes sans ESPT, de développer un usage nocif de ou une dépendance à l'alcool, et près de trois fois plus de risques de développer une dépendance à d'autres substances psychoactives. De plus, les femmes atteintes d'un ESPT couraient 2,5 fois plus de risques, par rapport aux femmes sans ESPT, d'avoir un trouble de dépendance à l'alcool et plus de quatre fois plus de risques de recevoir un diagnostic de dépendance à une autre substance psychoactive.

La relation comorbide entre l'ESPT et la dépendance à des substances psychoactives est source de spéculations et de débat depuis des années, de nombreuses personnes posant la question de ce qui se développe en premier, l'ESPT ou la dépendance à une substance psychoactive. Bien sûr, la question se pose différemment pour chaque individu qui possède son propre trauma, sa propre histoire et ses propres mécanismes pour y faire face. Plusieurs modèles théoriques ont été élaborés afin de contribuer à une meilleure compréhension de la relation complexe entre l'ESPT et la dépendance à une substance.

\section{Modèles théoriques pour la comorbidité entre l'état de stress post-traumatique et le trouble de dépendance à des substances psychoactives}

L'hypothèse de l'automédication postule que l'ESPT se développe en premier, étant donc le diagnostic principal, et que les substances chimiques sont employées pour atténuer ou pour apporter un soulagement des symptômes et de la détresse associés (Cash, 2006). La littérature sur le diagnostic double suggère que les formes particulières de substances utilisées par les personnes atteintes d'un ESPT varient fortement et pourraient dépendre du groupe de symptômes d'ESPT le plus prévalent et invalidant pour l'individu - par exemple, les personnes éprouvant surtout des symptômes d'évitement pourraient recourir principalement à des stimulants du système nerveux central qui améliorent la socialisation et la motivation, tandis que celles qui manifestent des symptômes d'hyperactivation neurovégétative pourraient retenir préférentiellement des sédatifs (Hien et coll., 2010 ; Saladin, Brady, Dansky \& Kilpatrick, 1995).

L'hypothèse du risque élevé considère que les individus qui recourent à l'alcool et à des substances chimiques mènent des vies qui sont plus risquées et par conséquent se retrouvent plus souvent exposées à des situations traumatiques ou stressantes (Cash, 2006 ; Jacobsen, Southwick \& Kosten, 2001).

L'hypothèse de la susceptibilité, néanmoins, postule que l'usage de drogues ou de l'alcool conduit un individu à être plus à risque de développer un ESPT lorsqu'il est exposé à un événement traumatique en raison des effets psychologiques et physiques de la consommation excessive de ces substances (Riggs \& Foa, 2008). Certains ont suggéré que le recours à l'alcool et à d'autres substances psychoactives interfère avec le processus de récupération normale, inhibant le traitement du matériel traumatique et l'habituation à celui-ci (Brady \& Sinha, 2005).

L'ESPT et le trouble de dépendance à des substances psychoactives sont des maladies à forte incidence comportant des impacts négatifs à long terme sur le fonctionnement biopsychosocial et sur le bien-être de l'individu (Perez-Dandieu \& Tapia, 2014). À ce titre, le traitement des deux maladies est souvent compliqué, le chemin vers la guérison étant semé d'embûches et d'obstacles à facettes multiples.

\section{EMDR (désensibilisation et retraitement par les mouvements oculaires)}

L'EMDR (désensibilisation et retraitement par les mouvements oculaires) est un traitement de première ligne de l'ESPT, internationalement reconnu (American Psychiatric Association, 2013 ; Oren \& Solomon, 2012). L'EMDR permet à un réseau mnésique traumatique, confiné et isolé, d'être libéré, désensibilisé, traité et résolu. Le protocole de traitement EMDR en huit phases est guidé par le modèle de traitement adaptatif de l'information (TAI) de Shapiro qui propose que tous les êtres humains possèdent la capacité innée à intégrer et à assimiler naturellement de nombreux aspects d'une expérience (Shapiro, 2001).

Dans le cas de l'ESPT, toutefois, on pense que le système de traitement de l'information est déséquilibré, empêchant ainsi l'intégration de l'expérience en la mémoire sémantique. Par conséquent, le souvenir traumatique demeure vif et déformé. On pense que le traitement EMDR réactive le traitement naturel des informations grâce aux stimulations bilatérales (SBL ; Shapiro \& Maxfield, 2002). L’EMDR implique que le rescapé d'un traumatisme se relie à tous les éléments du réseau mnésique - sensoriels, cognitifs, somatiques et émotionnels, tout en suivant des stimulations bilatérales et en maintenant une attention double (Shapiro, 2001). À la fin de l'EMDR, le rescapé d'un traumatisme connait une diminution ou une disparition des symptômes typiques de l'ESPT, et la charge émotionnelle désagréable et les sensations 
somatiques négatives associées auront disparu. L’image traumatique qui autrefois était vive apparaît maintenant comme floue ou lointaine, le souvenir traumatique étant perçu et ressenti comme un événement passé plutôt qu'actuel. Ce " recalibrage » du cerveau et du corps provoque une diminution ou une cessation des symptômes typiques de l'ESPT, supprimant le sentiment persistant de danger qu'éprouve le rescapé d'un traumatisme, permettant ainsi une amélioration du fonctionnement général (Shapiro, 1995).

\section{Protocoles EMDR modifiés pour les addictions}

La reconnaissance croissante de la prévalence du trouble de dépendance à une substance psychoactive en comorbidité avec un ESPT, s'est accompagnée d'une volonté d'étendre ou de modifier des traitements existants afin de permettre de meilleurs résultats de rétablissement pour cette cohorte de patients.

Par exemple, Hase, Schallmayer et Sack (2008) ont développé le protocole Craving Extinguished (CravEx) centré sur le concept de la mémoire addictive (MA), ces souvenirs de craving (appétence insatiable) et de rechutes dans la consommation de drogues. La mémoire addictive contenant des souvenirs épisodiques de craving obsessionnel-compulsif correspond à une mémoire inadaptée selon le modèle TAI. Le CravEx emploie le niveau d'envie irrépressible (LOU : Level of Urge) plutôt que les unités subjectives de perturbation (SUD) en postulant que cette stratégie EMDR permettra de réduire ou de supprimer le craving pendant le traitement EMDR de la mémoire addictive chez certains patients (Hase, 2010). Dans l'étude contrôlée randomisée de Hase et coll. (2008), 34 patients alcooliques chroniques étaient affectées de manière randomisée à l'un des deux traitements : le traitement habituel $(\mathrm{TH})$ ou le TH + EMDR. Les résultats indiquaient que les personnes affectées au groupe $\mathrm{TH}+$ EMDR ont montré une diminution significative du craving à la fin du traitement et un mois après le traitement, avec cinq personnes maintenant une abstinence dans le groupe TH + EMDR par rapport à zéro dans le groupe TH.

En parallèle, le DeTUR (protocole de désensibilisation des déclencheurs et de retraitement de l'envie irrépressible de consommer) de Popky (2005) se fonde sur le modèle TAI et utilise les SBL pour révéler et traiter les traumas fondamentaux ou problématiques centrales qui constituent la cause sous-jacente de l'addiction. Le DeTur est un protocole basé sur le trauma, combinant les ressources internes des patients avec des soutiens externes, dont le modèle en 12 étapes, la thérapie cognitive comportementale (TCC) et le TFT (thought field therapy; Popky, 2010).

Omaha (1998) a développé le Chemotion, un protocole de traitement de la dépendance chimique qui emploie une technique de dialogue relevant de la Gestalt pour identifier les déficits de relations d'objet qui peuvent ensuite permettre de constituer les cibles d'un traitement EMDR. Bien qu'il n'existe pas d'étude publiée investiguant les protocoles Chemotion et DeTUR en particulier, Abel et O’Brien (2010) décrivent le cas d'une femme qui est restée abstinente pendant deux ans après un traitement combinant les protocoles développés par Popky (2005) et Hase et coll. (2008).

Le protocole de l'état ressenti pour les addictions (FSAP : Feeling-State Addiction Protocol) de Robert Miller se distingue quelque peu des approches décrites jusqu'ici. Ses fondements reposent sur la théorie des états ressentis des addictions comportementales et aux substances psychoactives de Miller, qui postule que les addictions se créent quand des ressentis (sensation + émotion + cognition) deviennent rigidement liés à des objets ou comportements particuliers. Ce lien entre le ressenti et le comportement est un état ressenti et c'est l'état ressenti qui est conçu comme le moteur des envies irrépressibles et du craving associés à l'addiction, qu'elle soit comportementale ou liée à une substance (R. Miller, 2010). Bref, le FSAP combine la théorie des états ressentis des addictions comportementales et aux substances psychoactives avec une forme modifiée de l'EMDR. La différence entre le FSAP et les autres approches est que l'abstinence n'est ni nécessaire ni désirée. Au contraire, le FSAP requiert que les ressentis positifs associés à la consommation de l'alcool ou d'une autre substance psychoactive soient éprouvés aussi intensément que possible pendant le traitement, permettant ainsi l'élimination de l'état ressenti, éradiquant ainsi les cognitions négatives jusque-là " résistantes au changement " qui servaient à justifier les comportements compulsifs / addictifs (R. Miller, 2012). D’après Miller (2010), la modification la plus importante du protocole EMDR est l'approche utilisée dans le traitement des croyances négatives et de l'installation des croyances positives ; par exemple, lorsque l'état ressenti associé au comportement addictif a été traité, les croyances négatives qui soustendent l'état ressenti sont identifiées et les croyances positives désirées choisies.

\section{Procédures EMDR standard pour les addictions}

Il n'existe pas d'étude publiée sur l'effet des seules procédures EMDR standard sur la dépendance à l'alcool 
ou à d'autres substances psychoactives chez des patients recevant un traitement de leurs symptômes d'ESPT. Ce qui a été documenté, c'est l'usage de protocoles modifiés tels que le CravEx (Hase, 2010), le DeTUR (Popky, 2005) et le Chemotion (Omaha, 1998) mis en œuvre spécialement pour les personnes ayant un diagnostic double d'ESPT et de dépendance à des substances psychoactives.

Plusieurs auteurs considèrent que plusieurs composants de l'EMDR comme le lieu sûr, le développement et installation de ressources, et le tissage à deux mains peuvent être intégrés au traitement des addictions lors de différentes phases (Zweben \& Yeary, 2006). D'autres pensent que l'EMDR standard peut faire partie d'un plan de traitement complet lorsqu'il est utilisé conjointement avec le programme en douze étapes (Marich, 2009).

De plus, l'étude de Marich (2009) portant sur une femme présentant plusieurs addictions décrit les bénéfices de l'utilisation de l'EMDR dans le cadre du processus de soins de suivi après la fin d'un programme ambulatoire de huit semaines (un modèle de facilitation en douze étapes). Quinze séances d'EMDR standard ont été réalisées au cours d'une période de neuf mois, comprenant cinq scénarios futurs. Après l'EMDR, la participante a rapporté 18 mois d'abstinence et une amélioration générale de son fonctionnement et de son bien-être (Marich, 2009).

\section{Thérapies cognitives comportementales pour l'état de stress post-traumatique et le trouble de dépendance à une substance psychoactive}

\section{Traitements intégrés sans exposition}

Les traitements intégrés sans exposition possèdent une approche centrée sur le présent et n'impliquent donc pas de revenir sur les souvenirs traumatiques (exposition par visualisation) ni de confronter des situations sûres mais anxiogènes de la vie réelle qui sont évitées par le patient (McCauley, Killeen, Gros, Brady \& Back, 2012). Le traitement se concentre sur les réponses du patient au trauma et sur l'impact des symptômes traumatiques sur le fonctionnement et le bien-être. En particulier, le traitement comprend une stabilisation renforcée, une amélioration des capacités de coping (aptitude à gérer, à faire face) afin de prévenir la consommation de substances psychoactives et de gérer les symptômes d'ESPT, une restructuration cognitive avec une attention portée aux pensées inadaptées associées à la consommation de substances psychoactives et aux symptômes traumatiques, une psychoéducation sur l'ESPT et la dépendance aux substances psychoactives et le développement de compétences de communication efficace pour bâtir des réseaux de soutien sains (Hien et coll., 2010). Un exemple de traitement intégré sans exposition est Seeking Safety (rechercher la sécurité), le traitement le plus rigoureusement étudié à ce jour pour l'ESPT et la dépendance aux substances psychoactives, et qui génère le plus souvent des résultats positifs au niveau de l'ESPT et de la dépendance aux substances psychoactives dans l'ensemble des études (Najavits \& Hien, 2013). D'autres exemples comprennent l'Addictions and Trauma Recovery Integrated Model (ATRIUM ; D. Miller \& Guidry, 2001), le Trauma Adaptive Recovery Group Education and Therapy (TARGET ; Frisman, Ford, Hsui-Ju, Mallon \& Chang, 2008) et le Trauma Recovery and Empowerment Model (TREM ; Harris, 1998) ; toutefois, il existe peu de soutien empirique concernant l'efficacité de ces protocoles au niveau de la production d'améliorations maintenues en ce qui concerne l'ESPT et la dépendance aux substances psychoactives.

\section{Programmes intégrés d'exposition}

Le traitement parallèle de l'ESPT et de la dépendance aux substances psychoactives à l'aide de l'exposition prolongée (COPE) est le premier traitement à combiner la TCC fondée sur les preuves pour la dépendance aux substances psychoactives avec les composants clés de l'exposition prolongée pour l'ESPT qui comprend l'exposition par visualisation et in vivo. Brady, Dansky, Back, Foa et Carroll (2001) ont utilisé le COPE dans une étude non contrôlée de développement de la psychothérapie dans laquelle 39 patients présentant une comorbidité entre l'ESPT et une dépendance à une substance psychoactive ont reçu seize séances de thérapie individuelle incluant de l'exposition in vivo (séances 6 à 15) et par visualisation (séances 7 à 15). Les patients qui ont participé à au moins dix séances complètes ont montré une amélioration significative des groupes de symptômes d'ESPT et une diminution de la consommation de cocaïne entre la sélection et la fin du traitement (Brady et coll., 2001).

De manière similaire, Mills et coll. (2012) ont récemment effectué un essai contrôlé randomisé comparant le COPE (13 séances individuelles de 90 minutes avec un psychologue clinicien) plus le $\mathrm{TH}$ avec le TH seul $(\mathrm{TH}=$ recours par les patients à tout type de traitement des dépendances aux substances psychoactives disponibles dans la région, y compris des services ambulatoires, d'hospitalisation, résidentiels et des interventions pharmacologiques). L'étude comprenait 103 participants avec un ESPT civil et une dépendance 
à des substances psychoactives, et les résultats indiquaient dans le groupe de traitement COPE, entre la sélection et le suivi après neuf mois, une amélioration de l'intensité des symptômes d'ESPT sans augmentation de la sévérité de la dépendance aux substances.

Bien sûr, les traitements d'exposition intégrés ne se limitent pas au COPE. La thérapie de la dépendance à des substances psychoactives et de l'état de stress posttraumatique (SDPT) a été développée par Triffleman et ses collègues (Triffleman, 2000 ; Triffleman, Carroll \& Kellogg, 1999) et est réalisée sous forme d'une intervention de cinq mois comportant deux séances par semaine. La SDPT est une approche en deux phases qui comprend l'intégration de la TCC pour la dépendance à des substances psychoactives avec une exposition in vivo pour l'ESPT. La recherche concernant l'efficacité de cette thérapie est néanmoins limitée.

Dans cet article, nous présentons les données recueillies auprès de quatre patients suivis à notre clinique Post Traumatic Stress (PTS) à Fremantle en Australie-Occidentale, principalement en vue d'un traitement EMDR à la suite d'un diagnostic d'ESPT. De manière plus importante, notre article souligne l'effet du protocole EMDR standard de Shapiro (1995) sur la dépendance à l'alcool et à d'autres substances psychoactives immédiatement après la thérapie et lors du suivi après douze mois chez ce groupe de patients.

\section{Méthode}

L'étude a été menée à la clinique PTS de l'hôpital Fremantle, une clinique de recherche située dans un hôpital public important situé dans un cadre urbain. La clinique PTS a ouvert ses portes en 2009, est financée par l'université d'Australie-Occidentale et compte parmi ses salariés un psychiatre à temps partiel et une infirmière à temps partiel, tous deux ayant suivi une formation reconnue en EMDR.

\section{Participants}

Les participants étaient orientés vers la clinique PTS par des équipes de santé mentale, des médecins généralistes, des équipes médicales et des services d’aide sociale.

Ils étaient évalués à l'aide d'instruments de dépistage validés (voir le paragraphe sur les «Instruments de diagnostic et de dépistage ») ainsi que d'un entretien clinique complet.

Les critères d'inclusion pour l'admission à la clinique et au traitement étaient les suivants : un diagnostic d'ESPT fondé sur l'évaluation clinique, un score supérieur à 44 sur le questionnaire de l'ESPT (version civile [PCL-C] ; Blanchard, Jones- Alexander,
Buckley \& Forneris, 1996) et une stabilité affective et des compétences de tolérance au stress appropriées, appréciées en fonction de la présentation du patient au cours de l'entretien clinique, de ses antécédents et d'un score inférieur à 30 sur l'échelle des expériences dissociatives (Dissociative Experience Scale [DES] ; Bernstein \& Putnam, 1986).

Les patients étaient exclus de la participation au traitement s'ils avaient un diagnostic principal d'une maladie cérébrale organique importante, d'un trouble psychotique ou une idéation suicidaire active.

Avant le traitement, les patients donnaient leur consentement éclairé et recevaient des renseignements supplémentaires sur l'EMDR.

Des détails non essentiels ont été modifiés dans ce rapport afin de préserver l'anonymat des participants.

Sur les 31 patients orientés vers la clinique, 13 ne répondaient pas aux critères de traitement à la clinique. Sur les 18 patients acceptés pour le traitement, quatre satisfaisaient aux critères de l'ESPT et du trouble de dépendance à des substances psychoactives. Parmi ces quatre patients, trois (correspondant aux cas 2, 3 et 4) répondaient aux critères diagnostiques de la dépendance (présente) à l'alcool et un (correspondant au cas 1) à ceux de la dépendance présente à une substance psychoactive (marijuana). Les quatre patients ont commencé et terminé le traitement (donc pas d'abandon) et ont participé au suivi après douze mois.

Les antécédents des participants comprenaient une augmentation de la consommation d'alcool (cas 2, 3 et 4) et de l'utilisation d'une substance psychoactive (cas 1) après un événement traumatique. Les participants n'avaient pas cherché ni reçu de traitement antérieur pour leur dépendance à l'alcool ou à une substance psychoactive ; ils n'avaient pas non plus été orientés vers ni ne s'étaient activement engagés envers des services de réhabilitation des dépendances à l'alcool et aux substances psychoactives pendant la période où ils bénéficiaient d'une thérapie à la clinique PTS. Avant de commencer la thérapie EMDR, les participants recevaient des informations sur l'impact négatif que pouvait avoir l'utilisation continue de l'alcool et de substances psychoactives sur leur bien-être mental et physique ainsi que sur leur capacité à traiter efficacement et de manière efficiente le matériel traumatique pendant et après une séance d'EMDR.

Tous les participants ont déclaré ne pas avoir été exposés auparavant à une psychothérapie EMDR. Le cas 1 avait participé à douze séances de " thérapie par la parole " avec un thérapeute immédiatement après son traumatisme sur son lieu de travail. Aucun n'a pas participé à une psychothérapie concomitante pendant la période de son suivi en EMDR. Trois des quatre 
patients prenaient des psychotropes prescrits et suivis par le service ou le médecin référent.

Le traitement abordait principalement des traumas s'étant produits à l'âge adulte dans les quatre cas ; cependant, deux participants avaient également des antécédents de traumas dans l'enfance. Trois patients avaient vécu leur(s) trauma(s) dans un contexte professionnel, l'un comme policier (cas 4), l'autre comme pompier (cas 3) et le troisième comme gardien de prison (cas 2). Le cas 1 avait vécu son trauma en pratiquant un loisir aquatique.

Lorsque l'évaluation était terminée, les patients entamaient leur thérapie sans être placés sur liste d'attente. Le psychiatre et l'infirmière ont réalisé la psychothérapie EMDR en suivant le protocole standard ; ils étaient tous deux formés en EMDR par des formateurs accrédités. La psychothérapie EMDR se centrait uniquement sur les antécédents des patients qui concernaient leur ESPT et leurs traumas, pas sur leur dépendance à l'alcool ou à des substances psychoactives.

Il n'y avait pas de limite au nombre de séances de thérapie autorisées. Le nombre total de séances d'EMDR nécessaire pour chaque patient a varié de quatre à neuf. Le nombre moyen de séances EMDR nécessaires était de six.

\section{Instruments de diagnostic et de dépistage}

Mesures principales des résultats. Le Mini International Neuropsychiatric Interview (MINI) Plus (Sheehan et coll., 1997), un entretien diagnostique structuré, a été utilisé pour évaluer la dépendance à l'alcool et à d'autres substances psychoactives (au cours des douze derniers mois et en tout temps) et l'usage nocif de l'alcool et d'autres substances psychoactives (au cours des douze derniers mois et en tout temps). Suivant l'objectif de cet article et afin de souligner l'effet positif de l'EMDR sur la diminution de la consommation d'alcool et d'autres substances psychoactives, les données présentées seront centrées sur la dépendance et l'usage nocif présents (au cours des douze derniers mois) à l'alcool et à d'autres substances psychoactives.

En administrant le MINI Plus, le psychothérapeute pose une série de questions en lien avec chaque trouble du DSM-IV-TR (Diagnostic and Statistical Manual of Mental Disorders, $4^{\mathrm{e}}$ éd., texte révisé ; American Psychiatric Association, 2000). Pour obtenir un diagnostic de dépendance à l'alcool ou à d'autres substances psychoactives, le patient doit répondre " oui » à trois questions ou plus. Un exemple de question de dépistage d'une dépendance à l'alcool est : «Au cours des douze derniers mois, avez-vous eu besoin de boire plus afin d'obtenir le même effet que celui que vous obteniez quand vous avez commencé à boire la première fois? " (Sheehan et coll., 1997).

L'utilisation du MINI Plus reflétait notre objectif de réaliser un dépistage large de conditions comorbides car il est conçu pour évaluer et diagnostiquer 23 troubles psychiatriques. Il s'agit d'un outil de dépistage très sensible et précis qui peut être adapté à de nombreuses situations cliniques et de recherche ; il possède une excellente fiabilité et validité (Lecrubier, Sheehan \& Weiller, 1997).

Lors de l'évaluation initiale, trois participants (les cas 2, 3 et 4) répondaient aux critères du diagnostic de la dépendance présente à l'alcool selon le DSM-IV et un participant (le cas 1) obtenait le diagnostic de la dépendance présente à une autre substance psychoactive selon le DSM-IV. Les participants étaient de nouveau évalués après le traitement EMDR et douze mois après la fin du traitement.

Le PCL-C est un bref questionnaire d'autoévaluation utilisé pour évaluer les symptômes de reviviscence, d'évitement et d'hyperactivation neurovégétative de l'ESPT (Blanchard et coll., 1996). Il est fréquemment utilisé dans un cadre clinique pour suivre les symptômes et en tant qu'outil diagnostique dans les études de recherche. Comme le PCL-C ne permet pas aux investigateurs de confirmer que l'événement traumatique correspondant au critère $\mathrm{A} d u$ diagnostic d'ESPT du DSM-IV, il est davantage utile lorsqu'il est suivi d'un test diagnostique de deuxième niveau (comme le MINI Plus ; McDonald \& Calhoun, 2010). Lors de l'évaluation initiale, trois des quatre participants obtenaient un score supérieur au seuil de 44 pour un diagnostic d'ESPT, avec un score moyen de 55,25 .

Instrument de dépistage pour l'indication de l'EMDR. Le DES ou échelle des expériences dissociatives a été développé par Bernstein et Putnam (1986) afin d'évaluer le degré auquel les personnes éprouvent de la dissociation. Van Ijzendoorn et Schuengel (1996) considèrent que le DES possède une validité prédictive impressionnante, en particulier en ce qui concerne les troubles dissociatifs et les expériences traumatiques. De plus, addition, Carlson et coll. (1993) ont montré que le DES est un outil fiable et valide pour mesurer la dissociation dans plusieurs groupes et qu'il possède une bonne validité concomitante pour détecter des troubles dissociatifs sévères. Le DES a été administré pendant la phase d'évaluation seulement parce qu'il servait à identifier les personnes à risque de dissociation et pour lesquelles l'EMDR ne serait pas indiqué. 


\section{Procédure}

Chaque participant participait à une séance hebdomadaire d'EMDR en suivant le protocole standard en huit phases de Shapiro (1995). L'EMDR se centrait sur la résolution des souvenirs traumatiques particuliers du patient ; ainsi, il n'y a eu aucune adaptation ou modification du processus EMDR ; aucun souvenir addictif n'a été ciblé pendant la thérapie, quelle qu'en soit l'étape. En suivant le modèle TAI, des SBL ont été utilisées pour libérer, traiter et désensibiliser du matériel traumatique stocké de manière dysfonctionnelle. Le patient maintenait une attention double en se connectant à puis en intégrant les composants cognitifs, somatiques et émotionnels du souvenir traumatique.

\section{Présentations de cas}

Cas 1

Présentation. WC est un homme blanc de 28 ans, un électricien indépendant, vivant avec sa compagne depuis 9 ans.

Il a été orienté vers la clinique PTS par un médecin du centre hyperbare où il recevait une thérapie hyperbare pour des complications physiques (déficits de la démarche et de l'équilibre, troubles des sphincters et rétention urinaire) suite à un accident de plongée qui s'est produit quatre mois auparavant. Bien qu'il se soit rétabli physiquement, il éprouvait des symptômes évocateurs de l'ESPT. Il a été orienté pour une confirmation du diagnostic et pour déterminer l'indication d'une psychothérapie EMDR.

Antécédents du patient. WC se décrivait comme un plongeur de bon niveau, possédant une dizaine d'années d'expérience, notamment comme formateur de plongée auprès d'une entreprise de tourisme. Il dit que le jour de l'accident, il n'avait pas trop envie de plonger mais il pensait que cela réduirait son stress, compte tenu de la charge de travail et de la pression accrus qu'il connaissait depuis peu sur le plan professionnel. WC avait emprunté l'équipement de son beau-frère, car son propre équipement avait besoin d’être réparé, mais il n'était pas habitué à la bouteille de plongée en aluminium, la sienne étant en acier. Il décrit qu'il a plongé seul, ignorant les directives de sécurité recommandées et habituelles de toujours plonger avec une autre personne. Il se rappelle avoir perdu sa ceinture lestée et d'être par conséquent remonté rapidement, particulièrement au cours des cinq derniers mètres, jusqu'à la surface. Il décrit la peur comme un engourdissement et une perte de sensation qui se répandait dans ses extrémités supérieures et inférieures. Il était incapable d'appeler à l'aide ou d'activer l'engin de flottaison. Il se souvient du poids de la bouteille de plongée qui le plaçait en position latérale et de l'eau salée qui lui remplissait la bouche. À ce moment-là, il pensait qu'il allait mourir. Ayant été aperçu par d'autres plongeurs, il a pu être sorti de l'eau mais en raison d'une conscience altérée et d'une perte de connaissance, il ne se rappelle que des fragments du trajet de 45 minutes en bateau jusqu'à la terre ferme puis du transport vers les urgences. Au moment de l'évaluation, WC indique qu'il est troublé par des images perturbantes et par des souvenirs de l'accident de plongée, par des rêves et des cauchemars (de l'accident de plongée lui-même ainsi que de requins, de prédateurs de l'océan), par des accès de colère et par une tendance à redouter les intentions d'autrui. Depuis l'accident de plongée, WC avait augmenté sa consommation de marijuana, fumant un joint trois à quatre fois par semaine, le plus souvent seul ; ceci était une augmentation par rapport à un usage social occasionnel. Il pensait que la marijuana " aide à atténuer les choses . . . ça me calme ». Il rapportait également des événements traumatiques passés comprenant un incident de violences sexuelles dans l'enfance (VSE) et du harcèlement à l'école primaire. Il n'avait reçu aucun traitement antérieur de ses symptômes d'ESPT ou de sa consommation accrue de marijuana.

Évaluation initiale. Le MINI Plus indiquait que WC répondait aux critères pour l'ESPT et pour la dépendance (présente) à une substance psychoactive. Son score sur le PCL-C était de 46/85.

Traitement. Le plan initial consistait en des séances hebdomadaires d'EMDR mais en raison de sa charge de travail, la fréquence était deux fois par mois ; WC a eu besoin de cinq séances de thérapie EMDR. Il a identifié ses objectifs thérapeutiques comme "apprendre à mieux gérer mes émotions suivant l'accident de plongée. Mieux comprendre ce qui se passe dans mon cerveau. "

$1^{r e}$ séance d'EMDR.

Image cible : «Couché à la surface de l’eau, avec le goût de l'eau salée»

Cognition négative : «C’est ma faute »

Cognition positive : "J'ai de la chance d'être en vie», VoC (validité de la cognition) $=3 / 7$

Émotion : peur et colère, SUD $=6 / 10$, " frissons de froid dans mon corps ".

Les SBL étaient administrées par un engin pulsateur (TheraTappers) et des images ont émergé en lien avec les urgences, son admission à l'hôpital et 
la chambre de décompression. Son SUD s'est réduit à $4 / 10$.

WC a été convié à revenir à l'image cible et des SBL supplémentaires ont permis à l'accident de plongée lui-même d'être traité avec succès, avec l'émergence spontanée d'une position cognitive nouvelle : «Je suis plus fort maintenant . . . je m’en suis sorti » qui a été installée avec un VoC de 7/7. Le scanner corporel à la fin de la séance n'indiquait aucune résistance à cette croyance, le SUD était de 1/10 et WC a quitté la séance de manière calme.

$2^{e}$ et $3^{e}$ séances d'EMDR. La $2^{\mathrm{e}}$ séance d'EMDR s'est centrée sur l'accident de plongée, puisqu'une réévaluation de l'image cible donnait un SUD de 3/10. L'image cible était légèrement différente et focalisée sur la remontée rapide de WC avec " je suis incompétent ... j'aurais pu mourir . . . c'est ma faute ». Il éprouvait de la colère, située au niveau de l'estomac, le SUD étant maintenant à $6 / 10$. Des SBL à l'aide de l'engin pulsateur ont fait baisser le SUD à 3/10. Des tissages cognitifs concernant la responsabilité : «Comment auriez-vous pu savoir que la ceinture lestée aller se détacher et s'égarer ? Si cela arrivait à votre sœur, penseriez-vous que ce serait sa faute ? " ont permis de réduire encore le SUD. La cognition positive : «Ce n'est définitivement pas ma faute . . . j’ai survécu » a été installée avec un VoC de $7 / 7$. La $3^{\mathrm{e}}$ séance d'EMDR ciblait l'épisode de VSE, WC étant âgé de treize ans et son oncle étant l'agresseur. Les $4^{\mathrm{e}}$ et $5^{\mathrm{e}}$ séances ont ciblé des problématiques actuelles liées à l'estime de soi et des souvenirs concernant du harcèlement dans l'enfance qui les alimentaient. Un scénario futur a également été utilisé pendant la $5^{\mathrm{e}}$ séance, WC imaginant une expérience future dans laquelle il affrontait un client difficile par lequel il pouvait se sentir harcelé. WC n'a éprouvé qu'une perturbation légère et avec le retraitement, des interactions et des expériences positives ont émergé. Une réévaluation de toutes les images cibles et des souvenirs traumatiques a montré qu'ils paraissaient maintenant lointains et flous, sans croyance négative ni de charge émotionnelle associées.

La dépendance aux substances psychoactives de WC (passée, présente ou future) n’a jamais été ciblée pendant les séances d'EMDR. Aucun problème de croissance de la consommation de la substance psychoactive ne s'est produit pendant la thérapie EMDR.

Suivi. WC a bénéficié d'un suivi deux semaines après la fin de la thérapie, puis six mois et douze mois après l'EMDR. WC savait qu'il pouvait contacter la clinique PTS à tout moment s'il en éprouvait le besoin. Il se disait " libéré de tous les symptômes d'ESPT ". Il avait demandé à sa conjointe de l'épouser et gérait mieux ses clients et son personnel. Des raisons médicales l'empêchaient de reprendre la plongée mais il consacrait ses week-ends au surf et ne remarquait pas de déclencheur associé. Il n'avait consommé de la marijuana qu'à une seule occasion, dans un contexte de socialisation, depuis la fin de sa thérapie EMDR. Il n'éprouvait plus le besoin d'en consommer ni ne comptait sur elle pour réduire son anxiété. Le MINI Plus a confirmé ce qui précède en indiquant que WC ne répondait plus aux critères de l'ESPT ni à ceux de la dépendance présente à une substance psychoactive. Il obtenait 17 / 85 sur le PCL-C (un score supérieur à 44 suggérant un diagnostic d'ESPT).

\section{Cas 2}

Présentation. LK est une femme blanche divorcée de 37 ans, mère de deux filles âgées de cinq et de seize ans. Elle travaille à temps plein comme surveillante d'un établissement pénitentiaire pour mineurs (EPM), mais au moment de son orientation, elle était en arrêt maladie depuis deux semaines. Elle entretient depuis cinq mois une relation avec son compagnon Tom qui se montre soutenant.

LK a été orientée vers la clinique PTS par l'agent de service de l'hôpital psychiatrique local après qu'elle s'est présentée aux urgences pour le traitement de lacérations superficielles du poignet gauche en décrivant une difficulté à faire face suite à des événements traumatiques qui se sont produits pendant son enfance et à l'âge adulte.

Antécédents. LK rapportait plusieurs incidents traumatiques dont les premiers et les pires étaient des violences sexuelles de la part de son grand-père maternel entre les âges de six et de douze ans. Elle disait également avoir subi des violences de la part d'un homme inconnu à l'âge de douze ans, avoir été témoin de multiples épisodes de violences conjugales entre ses parents et avoir subi elle-même des violences conjugales dans son premier mariage. Son trauma le plus récent s'était produit deux ans auparavant et sur une période de deux mois pendant lesquels elle était traquée et menacée de manière répétée par d'anciens détenus de l'EPM où elle travaillait. LK décrivait comment d'anciens détenus avait obtenu accès à ses coordonnées personnelles, ce qui leur a permis, avec des membres de leurs familles, d'entrer par infraction chez LK en fin de soirée et de menacer de la violer et de la tuer ainsi que ses deux filles. Ceci avait amené LK à demander une ordonnance de protection. LK indiquait qu'elle n'avait pas éprouvé de symptômes d'ESPT avant ce dernier trauma, malgré ses antécédents traumatiques. 
Ses symptômes présents comprenaient des souvenirs intrusifs et des cauchemars concernant les VSE et l'introduction par effraction. Elle éprouvait des difficultés à s'endormir et à rester endormie. Elle était hypervigilante. Elle consommait en moyenne une demie bouteille de vin chaque soir depuis trois mois, avec des quantités encore plus importantes au cours des douze mois précédents. Elle avait réduit sa consommation d'alcool parce qu'elle connaissait ses effets négatifs sur son humeur et sa relation avec ses filles. Elle avait sollicité l'aide d'amis afin d'obtenir davantage de soutien émotionnel pendant cette période. Elle n'avait pas cherché de traitement formel pour son usage présent de l'alcool ni pour ses symptômes d'ESPT.

Évaluation initiale. Le MINI Plus indiquait que LK répondait aux critères de l'ESPT et de la dépendance présente à l'école. Son score sur le PCL-C était de $71 / 85$.

Traitement. Objectif thérapeutique : "Me sentir plus heureuse au jour le jour, ne plus faire de mauvais rêves, mieux dormir, être débarrassée des flash-backs et des cauchemars. »

La première séance d'EMDR de LK s'est centrée sur l'atroce violation du domicile, l'image cible étant un gros plan sur le visage de l'un des hommes, sa voix rageuse et son expression de colère. "Je suis en danger " était automatiquement exprimé comme cognition négative, la peur était située dans la poitrine et dans la gorge de LK et son SUD était à 9/10. "Je suis en sécurité » était sa cognition positive ; cependant, elle atteignait un VoC de 2/7. Les SBL ont démarré à l'aide d'un engin pulsateur (TheraTappers) et l'activation émotionnelle et physique de LK ont diminué tout au long de la séance. Son SUD s'est réduit à $1 / 10$ et comme elle l'a dit : "Il ne peuvent pas m'atteindre maintenant ... je suis en sécurité " (VoC 7/7). Le scanner corporel n'indiquait aucune résistance à cette croyance ni aucun inconfort physique résiduel.

La $2^{\mathrm{e}}$ séance a commencé par une réévaluation de la violation du domicile et LK indiquait que l'image cible était maintenant floue et que « tout ça ne semble plus me perturber du tout ». Selon l'EMDR standard de Shapiro et après une longue discussion avec LK, il a été décidé que le premier et pire souvenir traumatique serait ciblé pendant cette séance d'EMDR. LK a indiqué que l'image qui pouvait le mieux représenter le pire souvenir de VSE a eu lieu quand elle avait huit ans et qu'elle était seule avec son grand-père dans sa salle de bain. Sa cognition négative "je suis mauvaise " s'accompagnait d'émotions de peur et de honte qu'elle ressentait " partout » avec un SUD à 10/10. Sa cognition positive, «je n'étais qu'une enfant » avait un VoC de $2 / 7$ seulement.

Le thérapeute a démarré les SBL à l'aide d'un engin pulsateur ; après deux séries, LK est devenue inexpressive et distante. Le thérapeute l'a aidée à revenir au présent à l'aide de techniques d'ancrage et d'exercices de respiration lente. LK est " allée dans son lieu sûr » et après cinq minutes elle se sentait « de nouveau ellemême ». Elle avait hâte de recommencer l'EMDR. Pour éviter que le même phénomène se reproduise, le thérapeute a consacré du temps à l'installation de personnages ressources et a altéré l'image cible de sorte qu'elle apparaisse comme une photo en noir et blanc. LK a pu traiter cette cible et d'autres images et souvenirs associés de VSE. Son SUD diminuait régulièrement pour terminer à 1 et le $\mathrm{VoC}$ de sa cognition positive était à $7 / 7$.

LK a participé à des séances hebdomadaires de psychothérapie EMDR, nécessitant quatre séances en tout. En plus des VSE et de la violation du domicile, elle a ciblé la colère qu'elle éprouvait actuellement envers sa mère «car elle ne m’a pas protégée des violences ». Après le traitement, la cognition positive «ce n'est pas sa faute . . e elle a fait de son mieux " a émergé spontanément. Enfin, un travail sur des scénarios futurs a été réalisé pour cibler l'anxiété anticipatrice qu'elle éprouvait à l'idée de reprendre ses fonctions professionnelles et de croiser un ancien détenu dans la rue.

Chaque semaine, le thérapeute reprenait l'image cible déjà traitée et chaque fois, LK indiquait que l'image était distante et floue, sans connotation émotionnelle. Elle a décrit une diminution de ses symptômes d'ESPT et une amélioration de son fonctionnement en général. Elle rapportait qu'elle n'avait plus besoin d'alcool pour amoindrir ses symptômes et que la quantité d'alcool qu'elle consommait s'était par conséquent considérablement réduit. Sa dépendance à l'alcool n'a jamais été ciblée pendant les séances d'EMDR.

Suivi. Bien que LK n'ait eu besoin que de quatre séances de psychothérapie EMDR, elle a été suivie deux semaines après la fin de la thérapie puis six mois et douze mois plus tard. Elle savait qu'elle pouvait contacter la clinique PTS à tout moment si elle avait besoin de soutien ou d'une thérapie supplémentaire.

Le suivi après douze mois a montré qu'elle avait maintenu son amélioration sans aucun retour de ses symptômes d'ESPT. Elle indiquait qu'elle n'éprouvait plus d'envie irrépressible d'alcool ni n'avait bu d'alcool en étant seule depuis le traitement. Elle rapportait qu'elle buvait de l'alcool dans des situations sociales, 
en moyenne une fois par quinzaine, consommant une ou deux doses standard, sans épisode d'alcoolisation excessive ni d'intoxication. Elle a repris ses fonctions professionnelles. L'entretien clinique et les résultats du MINI Plus et du PCL-C (21/85) indiquaient qu'elle ne répondait plus aux critères d'une dépendance présente à l'alcool ni à ceux de l'ESPT.

\section{Cas 3}

Présentation. RC est un homme australien de 47 ans, marié et père de deux enfants adolescents. Il vit dans sa maison familiale en banlieue avec sa femme depuis 27 ans. Son psychiatre l'a orienté vers la clinique PTS pour la confirmation de son diagnostic d'ESPT et pour évaluer l'indication d'une psychothérapie EMDR. RC était en arrêt maladie depuis quatre mois, étant incapable d'assumer son poste dans l'équipe de sauvetage par hélicoptère de la police.

Antécédents. RC rapporte qu'il a été exposé à de multiples traumas dans le cadre de son travail dans la police, plus particulièrement depuis qu'il a rejoint l'équipe de sauvetage par hélicoptère de la police en 1991. Les événements perturbants les plus marquants comprenaient la récupération des corps de quatre individus décédés en mer et le crash de son hélicoptère alors qu'il effectuait une visite de routine auprès d'une école primaire à des fins pédagogiques. L'incident le plus perturbant de tous s'était produit 14 ans auparavant : il s’agissait de récupérer les corps de quatre collègues policiers ("ils étaient mes potes ») qui travaillaient également à l’équipe de sauvetage par hélicoptère de la police et qui étaient décédés lorsque leur hélicoptère a percuté une falaise. RC disait que ses symptômes d'ESPT avaient commencé un mois après cet événement. Il avait participé à douze séances de " thérapie par la parole " organisées par son employeur. Il y attribuait peu d'effets positifs. Il n'a pas évoqué ses symptômes d'ESPT à ses collègues ni à sa famille. Ces symptômes s'étaient peu à peu aggravés. Il fabriquait des excuses et évitait certaines activités au travail. Il a commencé à boire de l'alcool pour combattre son anxiété, pour l'aider à dormir et pour faire cesser les symptômes de reviviscences. Au moment de l'évaluation, il buvait de l'alcool cinq jours par semaine, commençant vers 15 heures et consommant en moyenne une bouteille de vin rouge $(750 \mathrm{ml})$, quatre "stubbies » (bouteilles de bière de $375 \mathrm{ml}$ ) et deux shooters de bourbon. Il buvait seul dans la cabane du jardin mais il disait accorder la priorité à ses responsabilités parentales/ familiales et domestiques. Il maintenait le lundi et le mardi comme des journées sans alcool en raison d'une activité de coach et des heures de sport de son fils. Il n'avait cherché aucun traitement ni aucune intervention ou thérapie pour sa dépendance à l'alcool, que ce soit récemment ou par le passé. Il ne souhaitait pas être orienté vers une cure de sevrage ou des services de réhabilitation.

Évaluation initiale. D’après le MINI Plus, RC répondait aux critères de l'ESPT et de la dépendance présente à l'alcool. Son score sur le PCL-C était de $42 / 85$.

Traitement. RC a participé à six séances d'EMDR. La première séance a ciblé le souvenir le plus émotionnellement chargé : avoir récupéré et identifié les différentes parties des corps éparpillés des collègues décédés dans un accident d'hélicoptère. Malgré plusieurs tentatives et une longue exploration, RC avait du mal à identifier une cognition négative. Il décrivait une anxiété débordante sous la forme d'une sensation d'appréhension dans le ventre. Il évaluait son SUD à $3 / 10$. Le thérapeute a immédiatement interrogé la validité et l'incongruence du SUD : comment une anxiété débordante pouvait-elle être éprouvée à un niveau si faible? RC a reconnu qu'il bloquait les sensations et les émotions associées, " un mécanisme protecteur . . . une habitude de la police ». Pour prévenir un tel blocage, le thérapeute a pris du temps pour souligner auprès de RC l'importance de se connecter à tous les éléments du réseau mnésique afin d'assurer un traitement efficace et pour que la résolution du souvenir traumatique puisse ainsi se produire. Le thérapeute a encouragé RC à se relier à tous les composants visuels, tactiles, auditifs et olfactifs de l'image du trauma ; cette fois, RC a annoncé un SUD à 8/10. Des SBL sous forme de mouvements oculaires saccadiques ont eu lieu pendant 50 minutes. Plusieurs images d'expériences professionnelles traumatiques passées ont émergé : la récupération de corps en mer, l'accident d'hélicoptère de RC et des incidents de violences conjugales. Le SUD de RC s'est réduit à 2/ 10 . Le temps de la séance étant presqu'écoulé, l'exercice du lieu sûr a été utilisé pour terminer.

La deuxième séance a commencé par la réévaluation de l'image cible de la première cible. RC a indiqué qu'elle paraissait assez distante et qu'il la ressentait comme telle également. Il a décrit une anxiété résiduelle qu'il évaluait à 4 / 10, située au niveau du ventre, avec la cognition négative "Je n'ai pas le contrôle . . . je suis faible ". De nouveau, des SBL sous forme de mouvements oculaires saccadiques ont été utilisées, traitant l'image cible avec succès, réduisant le SUD à $1 / 10$, avec une cognition positive « je le surmonterai » renforcée de $2 / 7$ à $6 / 7$. 
À la troisième séance, $\mathrm{RC}$ a décrit une image « qui m’a perturbé cette semaine ". Il décrivait un moment pendant la récupération des parties du corps de son collègue, où il était hélitreuillé avec une housse mortuaire. La housse mortuaire s'est déchirée et un os du défunt a frôlé la jambe de RC. Pendant la séance, il indiquait ressentir l'odeur du sang et de la chair, ce qui le rendait nauséeux et anxieux. Par conséquent, le thérapeute a consacré la séance au traitement de cette image et de la cognition négative associée « je ne suis pas en sécurité . . . la mort est partout autour de moi ». Son score SUD était à $9 / 10$, situé au niveau du ventre. RC était ébranlé pendant la séance, disant à un moment " je crois que je vais peut-être vomir ". Malgré cela, le traitement s'est bien poursuivi, son SUD s'est réduit, atteignant $1 / 10$ et une cognition positive ressentie comme complètement vraie, « je vais bien maintenant ».

Après trois séances d'EMDR, RC indiquait une diminution de ses attaques d'angoisse de six à trois par jour, moins d'effroi, plus d'activités sociales dont des sorties spontanées au restaurant et une diminution de ses symptômes de reviviscences. Son envie forte et son désir de consommer de l'alcool avaient légèrement baissé.

Les séances 4 et 5 ont ciblé des déclencheurs présents : un sac de courses lourd rebondissant contre sa jambe, déclenchant une sensation similaire à la housse mortuaire déchirée qui l'avait frôlé ; une sortie à la plage et l'odeur des algues déclenchant l'odeur de chair morte.

La sixième séance a abordé son anxiété concernant son retour au travail, sa capacité de fonctionner à son poste et la manière de gérer des traumas similaires s'il y était confronté. La dernière séance a également permis de réévaluer toutes les cibles traitées au cours de la thérapie. RC rapportait que les images semblaient maintenant floues et moins vives, " elles ne me prennent plus la tête».

La dépendance à l'alcool de RC n'a jamais été ciblée pendant sa psychothérapie EMDR.

Suivi. RC a participé à un entretien de suivi à la clinique PTS deux semaines après la fin de la thérapie puis de nouveau six et douze mois plus tard. Il savait qu'il pouvait contacter la clinique PTS à tout moment s'il éprouvait le besoin d'obtenir du soutien ou de poursuivre sa thérapie. Il a continué à voir son psychiatre.

Lors du suivi après douze mois, même s'il répondait toujours aux critères de la dépendance à l'alcool sur le MINI Plus, RC rapportait que son craving et que la quantité d'alcool consommée avaient diminué depuis la thérapie EMDR. Il indiquait également une réduction du nombre de jours par semaine où il buvait de l'alcool (de cinq à trois) et disait ne plus mélanger l'alcool fort, le vin et la bière en buvant. Il continue à refuser l'orientation vers des services de réhabilitation et de désintoxication. L'amélioration de ses symptômes d'ESPT s'est maintenue avec un score de 29/85 sur le PCL-C (un score supérieur à 44 reflète un diagnostic d'ESPT). Il n'avait pas repris son poste de policier mais envisageait une réorientation professionnelle et plus particulièrement d'acquérir une entreprise de services de jardinage en franchise. Il se disait toujours heureux avec son épouse et a commencé à pratiquer le water-polo en équipe de vétérans.

Cas 4

Présentation. SK est un homme blanc de 53 ans, travaillant à temps plein comme pompier. Il vit depuis dix ans avec sa compagne soutenante, Lisa, et a deux enfants adultes d'un premier mariage. Il a été orienté vers la clinique PTS par son médecin généraliste après l'amplification de ses symptômes d'ESPT qui avaient fait leur première apparition deux ans auparavant et s'étaient aggravés au cours des douze derniers mois.

Antécédents. SK rapporte avoir été exposé à des traumas multiples depuis qu'il est devenu pompier en 1988. Il y a deux ans, des symptômes d'ESPT sont apparus, avec des flash-backs, des souvenirs intrusifs et des cauchemars au sujet de la récupération de personnes décédées dont le corps était souvent mutilé. Les symptômes s'étaient accrus par leur intensité et par leur fréquence au cours des douze derniers mois. SK recourait à l'alcool dans une tentative de réduire les symptômes intrusifs et d'hyperactivation. Il avait commencé à boire rapidement des quantités importantes d'alcool en début d'année. Il n'avait pas cherché ou suivi, ni été orienté vers une thérapie pour sa dépendance à l'alcool. Son employeur l'avait orienté vers un conseiller de Crisis Care $^{1}$; il a bénéficié de quatre séances en tout.

Évaluation initiale. Le MINI Plus indiquait que SK répondait aux critères de l'ESPT et de la dépendance présente à l'alcool. Son score sur le PCL-C était de $62 / 85$.

Traitement. SK a bénéficié de six séances d'EMDR. Son objectif thérapeutique était : «empêcher les pensées et les images d'être si intrusives . . . avoir plus de contrôle ».

${ }^{1} \mathrm{NDT}$ : plateforme téléphonique d'écoute et d'aide d'urgence, disponible à toute heure. 


\section{Séance d'EMDR 1}

Image cible : « M'occuper d'un accident de la voie publique, la conductrice décédée était policière, la voiture était pliée autour d’un arbre, le corps écrasé, la pluie, il faisait froid et sombre, le bruissement des feuilles dans les arbres, le vent, la voiture presque coupée en deux ".

$\mathrm{CN}$ : «Je suis démuni »

$\mathrm{CP}$ : «J'ai fait de mon mieux»; VoC : $2 / 7$

Émotion : «peur », SUD 8/10, dans la tête, les mains et la poitrine

Les stimulations bilatérales ont été administrées sous forme de mouvements oculaires et pendant le traitement, SK a noté qu'émergeaient plusieurs images différentes provenant de multiples accidents et tragédies auxquels il avait été confronté au fil des années. Ceci s'accompagnait d'une modification des sensations physiques (de la tête vers les épaules, puis vers le ventre, puis vers le dos) et des émotions (de la peur à l'anxiété, à la colère). Vers la fin de la séance, un changement spontané est apparu au niveau de la perception et des croyances de SK concernant son travail de pompier : «Ce boulot n'est pas si mal . . . on aide des gens ... on accomplit quelque chose ... je me sens mieux . . . l'image est toujours un peu là mais je me sens OK avec ça ". Son SUD avait diminué à 2/10 mais en raison des contraintes de temps, la cognition positive et le scanner corporel n'ont pas été abordés. Le thérapeute a terminé la séance avec le lieu sûr.

Séance d'EMDR 2. Cette séance a continué à traiter l'image de la policière décédée ; toutefois, l'image s'était légèrement modifiée depuis la semaine précédente - elle apparaissait maintenant comme un " arrêt sur image » et SK visualisait une serviette sur son visage. À la fin de la séance, le SUD de SK s'était réduit jusqu'à $1 / 10$ et la cognition positive " j'ai fait de mon mieux . . . la mortalité fait partie de mon boulot . . . j'ai une meilleure acceptation " a été pleinement installée (VoC $=7 / 7)$.

Séances d'EMDR 3 à 5 . Ces séances se sont centrées sur un autre accident tragique - le décès d'un garçon adolescent qui roulait à vélo et qui a été tué par une voiture en excès de vitesse conduite par deux jeunes en état d'ivresse. Comme SK en était ébranlé, le thérapeute l'a convié à modifier l'image pour la voir sous forme d'une photo à effet sépia et à distance (vue du ciel). À la fin de ces séances, le SUD de SK avait diminué de $7 / 10$ à $1 / 10$ et l'image initiale paraissait floue et distante.

Séances d'EMDR 6 à 8 . À la sixième séance, SK rapportait que toutes les cibles passées étaient floues, sans charge émotionnelle négative. Il décrivait un meilleur élan et une motivation accrue, son humeur s'était améliorée tout comme son sommeil, sans cauchemar depuis deux semaines. Il avait réduit sa consommation d'alcool sans autre intoxication massive. Cependant, il s'inquiétait de ce qu'il pouvait vivre à l'avenir dans le cadre de son travail et se demandait si et comment il gérerait des tragédies similaires. Le sentiment d'angoisse et les souvenirs qui l'alimentaient sont devenus la cible de ces séances d'EMDR. Le SUD s'est réduit de 6/10 à 1/10. L'installation de la cognition positive " même si je deviens anxieux, je peux le surmonter . . . j’ai déjà fait face . . . c'est normal d'être anxieux parfois ... je suis compétent ... je me sens plus fort » a suivi, atteignant un VoC de $6 / 7$.

Séance d'EMDR 9. Cette dernière séance s'est centrée sur l'anxiété anticipatrice résiduelle qu'éprouvait SK par intermittence vis-à-vis des interventions sur les lieux d'accidents à l'avenir. Dans les scénarios futurs, SK imaginaient des expériences futures au travail puis des expériences futures difficiles au travail, afin de réussir à réduire l'appréhension restante. La dépendance à l'alcool de SK n'a jamais été ciblée au cours de sa thérapie EMDR.

Suivi. SK a assisté à un suivi à la clinique PTS trois semaines après la fin de sa thérapie, puis six et douze mois plus tard. Le suivi après douze mois n'indiquait aucun signe de symptômes d'ESPT. Il rapportait que les images traumatiques conservaient leur forme altérée sans perturbation émotionnelle ou physiologique. Il planifiait de longues vacances avec sa compagne. Il décrivait une abstinence totale vis-à-vis de l'alcool depuis sa thérapie EMDR, sans éprouver de craving. Le MINI Plus indiquait qu'il ne répondait plus aux critères diagnostiques de l'ESPT ou de la dépendance à l'alcool. Il a obtenu 18/85 sur le PCL-C (un score supérieur à 44 indique la présence d'un ESPT).

\section{Résultats}

Comme ce n'était pas notre intention d'isoler ou de traiter une cohorte de patients atteints d'un ESPT et d'un trouble de dépendance à une substance, aucun instrument de dépistage validé de la dépendance à l'alcool ou à une autre substance n’a été employé lors du processus d'évaluation.

En revanche, les scores du MINI Plus et les déclarations des participants ont permis de souligner l'amélioration de la dépendance à l'alcool ou à une autre substance.

En résumé, l'évaluation réalisée douze mois postEMDR indiquait qu'un patient répondait aux critères diagnostiques de la dépendance présente à l'alcool et 
aucun patient ne répondait aux critères diagnostiques de la dépendance présente à une substance.

Le cas 1 disait avoir consommé de la marijuana une fois dans une situation sociale depuis la fin de son traitement. Il rapportait qu'il n'avait plus de craving pour la marijuana et n'en avait pas besoin pour soulager son anxiété. Le cas 2 rapportait ne plus avoir de craving pour l'alcool et ne pas en avoir consommé en étant seule depuis le traitement. Elle disait consommer de l'alcool dans des situations sociales, en moyenne une fois par quinzaine, en buvant un ou deux verres standard, sans épisode de consommation excessive ou d'intoxication. Le cas 3 , même s'il répondait encore aux critères d'une dépendance présente à l'alcool, rapportait que son craving vis-à-vis de l'alcool et le volume d'alcool consommé s'étaient réduits depuis le traitement. De plus, il rapportait une diminution du nombre de jours par semaine où il buvait de l'alcool (de cinq à trois jours) et ne plus mélanger l'alcool fort, le vin et la bière ces jours-là. Le cas 4 décrivait une abstinence totale de toute consommation d'alcool depuis le traitement, sans expérience de craving.

Les symptômes d'ESPT des participants se sont améliorés avec des scores moyens sur le PCL-C passant de 55,25 (un score supérieur à 44 reflétant un diagnostic d'ESPT) lors de l'évaluation initiale à 21,25 lors du suivi après douze mois (voir figure 1).

\section{Discussion}

La psychothérapie EMDR est un traitement psychologique de première ligne bien établi pour l'ESPT (American Psychiatric Association, 2013 ; Bisson et coll., 2007).

Il importe de noter que dans notre étude, les améliorations au niveau de la dépendance des patients à l'alcool et à d'autres substances se sont produites sans modifier le protocole EMDR standard ou mettre en œuvre des protocoles d'addictions (protocole DeTUR de Popky [2010], protocole CravEx de Hase [2010] ou protocole Chemotion d'Omaha [1998]) ou le FSAP de Robert Miller. De plus, aucune intervention pharmacologique pour les addictions ni aucune désintoxication en ambulatoire ou en résidentiel n'ont été requises.

En examinant les résultats ainsi que les déclarations des quatre participants lors du suivi après douze mois, il serait raisonnable de suggérer que l'hypothèse d'automédication est significative ici. Tous les participants rapportent un changement positif au niveau de leur consommation préférée d'alcool ou d'une autre substance après la psychothérapie EMDR et une diminution de leurs symptômes d'ESPT. En particulier, trois participants ont rapporté une réduction de leur craving et un participant a rapporté l'arrêt complet du craving depuis la fin de l'EMDR. De manière similaire, un participant a décrit une abstinence totale et trois participants

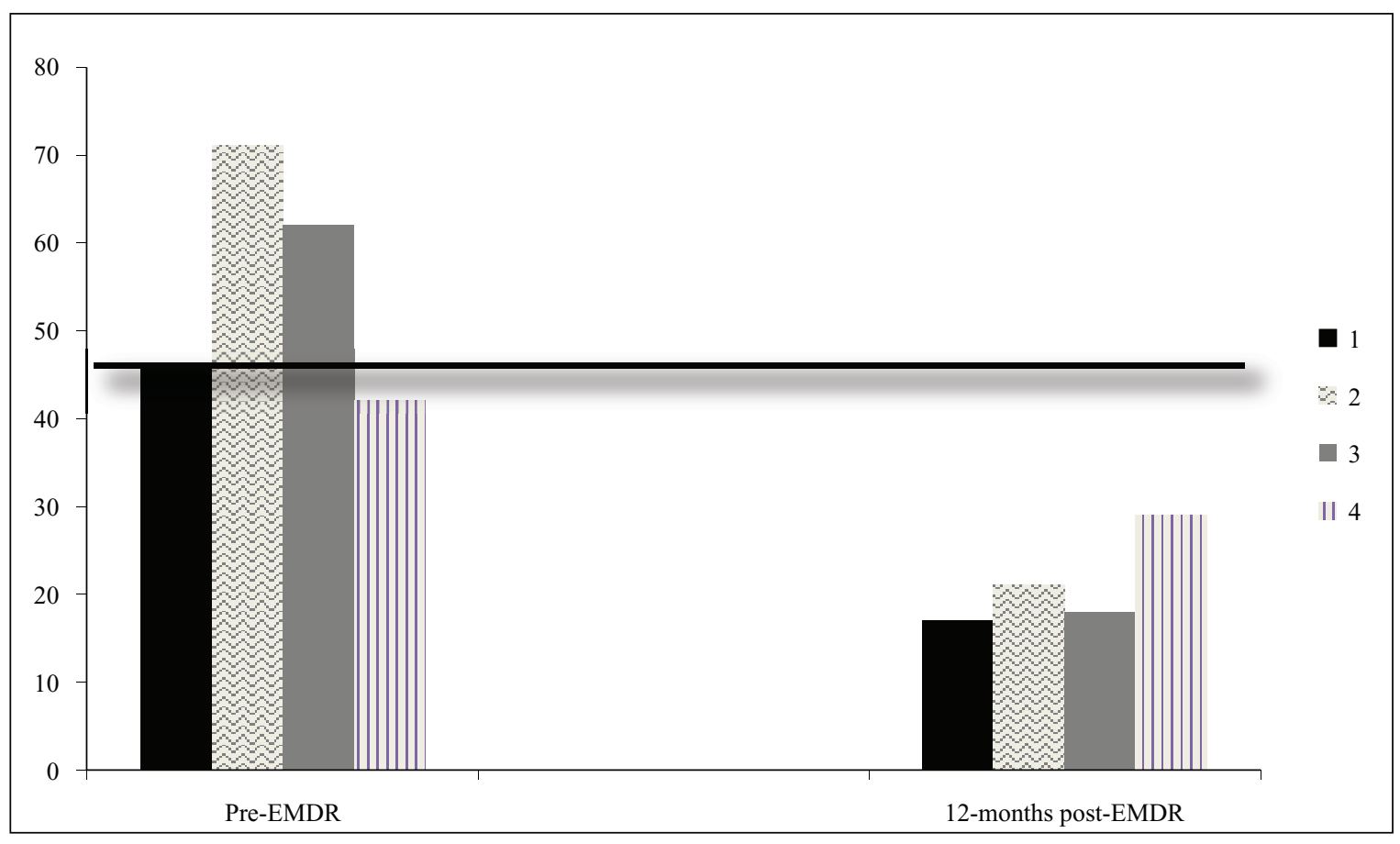

FIGURE 1. Scores de la liste de contrôle de l'ESPT (PCL-C) : pré-EMDR et suivi après douze mois. ESPT = état de stress post-traumatique

Note. Pre-EMDR $=$ pré-EMDR $; 12$-months post-EMDR $=12$ mois post-EMDR. 
ont décrit une diminution générale de la quantité d'alcool ou d'une autre substance consommée au cours des douze mois suivant l'EMDR. Deux des participants qui rapportaient une consommation post-EMDR ont identifié la consommation comme se produisant uniquement dans des situations sociales, sans épisode d'intoxication ou de consommation rapide et excessive.

Ceci amène à poser plusieurs questions : un traitement concomitant est-il toujours nécessaire pour les individus présentant à la fois un ESPT et un trouble de dépendance à une substance ? Est-il faisable/efficace d'entamer une psychothérapie EMDR avant de traiter l'addiction ? Pourquoi l'incidence de dépendance à l'alcool s'est-elle réduite dans la cohorte de patients décrite alors que seul un protocole EMDR standard a été employé?

On pourrait arguer que les réponses pourraient se trouver en lien avec le mécanisme d'action de l'EMDR. Selon Solomon et Shapiro (2008), l'EMDR permet à un réseau mnésique traumatique jusquelà isolé de se transformer d'un état épisodique à un état sémantique, permettant au souvenir traumatique traité d'être assimilé à d'autres réseaux mnésiques fonctionnels. Une fois traité, le trauma peut être considéré et ressenti comme appartenant au passé plutôt que comme un événement vif et présent, ce qui produit une diminution ou une disparition des symptômes d'ESPT.

De manière similaire, le stress chronique et la menace constante d'un danger perçu et réel peut comporter un effet nocif sur l'état biologique et neurologique du rescapé d'un trauma (Frodl \& O’Keane, 2013). On peut proposer que la suppression des symptômes d'ESPT parl'EMDR pourrait avoir pour résultat l'élimination de la suractivation de l'axe hypothalamo-hypophyso-surrénalien (HHS) et une réduction de la sécrétion prolongée et toxique de cortisol. De plus, l'ESPT est associé à des changements au niveau du débit sanguin, du métabolisme et du volume de matière grise dans des aires du cerveau limbique telles que l'amygdale et l'hippocampe (Pagani, Högberg, Fernandez \& Siracusano, 2013). On sait que l'amygdale est une structure importante pour la constitution de la mémoire épisodique et qu’elle serait fortement impliquée dans le conditionnement de la peur (Hayes, Hayes \& Mikedis, 2012 ; Woon, Sood \& Hedges, 2010). On peut donc proposer que le traitement du matériel traumatique qui se produit pendant l'EMDR résulte en une restabilisation de ces structures, permettant au corps de retrouver l'homéostasie en avançant vers la guérison. Globalement, la diminution des symptômes d'ESPT ainsi que la suppression $\mathrm{du}$ stress chronique pourrait expliquer le besoin réduit d'utiliser l'alcool ou des drogues pour inhiber des circuits cérébraux associés à l'anxiété et le besoin réduit de s'autoréguler/s'automédiquer à mesure que la lucidité et le fonctionnement s'améliorent. Compte tenu des résultats intéressants énoncés, il est raisonnable de suggérer que la recherche future sur l'impact de l'EMDR standard sur la dépendance à l'alcool et à d'autres substances serait bien justifiée. De manière similaire, tel qu'énoncé dans l'introduction, aucun des protocoles EMDR modifiés pour la dépendance à l'alcool ou à d'autres substances n'a été rigoureusement évalué ; de la recherche future serait nécessaire pour déterminer leur efficacité générale.

Les limites des données relèvent d'une taille d'échantillon relativement réduite et de l'absence d'un groupe de contrôle. Dans les études à venir, il pourrait être utile qu'un clinicien indépendant, non impliqué dans le traitement, réalise les évaluations et emploie un instrument validé propre à la dépendance aux substances, tel que l'Obsessive-Compulsive Drinking Scale (évaluant la consommation d'alcool obsessionnelle compulsive) (Anton, Moak \& Latham, 1995) ou le Munich Alcoholism Test (Feuerlein, Ringer, Küfner \& Antons, 1979). De manière similaire, les études futures, selon les besoins de la cohorte de participants et pour éviter une exacerbation ou des complications au niveau de la dépendance à une substance, devraient peut-être envisager une approche différente ou formelle pour contrôler ou traiter la consommation d'alcool ou d'autres substances au cours de la thérapie EMDR. Un examen systématique et une méta-analyse de Roberts, Roberts, Jones et Bisson (2015) indique l'existence de preuves selon lesquelles une intervention psychologique centrée sur le trauma, réalisée en présence d'un trouble de dépendance à une substance, peut réduire tant la sévérité de l'ESPT que la consommation d'alcool ou d'une autre substance. La revue de la littérature de Najavits et Hien (2013) identifie Seeking Safety (" rechercher la sécurité»), une thérapie TCC sans exposition, comme produisant le plus de résultats positifs et il s'agit du seul traitement qui a une meilleure performance que le contrôle pour à la fois l'ESPT et le trouble de dépendance à une substance. Comme nous l'avons énoncé dans notre introduction, il n'existe pas d'étude publiée qui porte précisément sur les protocoles Chemotion, DeTUR ou le FSAP de Robert Miller. De futures études investiguant l'impact de ces protocoles en comparaison avec le traitement habituel, la TCC sans exposition (comme Seeking Safety) et avec exposition seraient très bénéfiques.

En conclusion, notre étude suggère que le traitement de l'ESPT avec le protocole EMDR standard peut avoir un effet positif sur les symptômes de la 
dépendance à une substance jusqu'à douze mois au moins après le traitement. Des études contrôlées avec des groupes de taille supérieure sont nécessaires pour confirmer et étoffer ces résultats.

\section{Bibliographie}

Abel, N. J. \& O’Brien, J. M. (2010). EMDR treatment of comorbid PTSD and alcohol dependence: A case example. Journal of EMDR Practice and Research, 4(2), 50-59.

American Psychiatric Association. (2000). Diagnostic and statistical manual of mental disorders (4 éd., texte révisé). Washington, DC : Auteur.

American Psychiatric Association. (2013). Practical guidelines for the treatment of patients with acute stress disorder and posttraumatic stress disorder. Arlington, VA : Auteur.

Anton, R. F., Moak, D. H. \& Latham, P. (1995). The Obsessive-Compulsive Drinking Scale: A self-rated instrument for the quantification of thoughts about alcohol and drinking behavior. Alcoholism, Clinical and Experimental Research, 19(1), 92-99.

Bernstein, E. M. \& Putnam, F. (1986). Development, reliability, and validity of a dissociation scale. Journal of Nervous and Mental Disease, 174, 727-735.

Bisson, J. I., Ehlers, A., Matthews, R., Pilling, S., Richards, D. \& Turner, S. (2007). Psychological treatments for chronic post-traumatic stress disorder: Systematic review and meta-analysis. British Journal of Psychiatry, 190, 97-104.

Blanchard, E. B., Jones-Alexander, J., Buckley, T. C. \& Forneris C. A. (1996). Psychometric properties of the PTSD checklist (PCL). Behaviour Research and Therapy, 34, 669-673.

Brady, K. T., Dansky, B. S., Back, S. E., Foa, E. B. \& Car- roll, K. M. (2001). Exposure therapy in the treatment of PTSD among cocaine-dependent individuals: Preliminary findings. Journal of Substance Abuse Treatment, 21, 47-54.

Brady, K. T. \& Sinha, R. (2005). Co-occurring mental and substance use disorders: The neurological effects of chronic stress. American Journal of Psychiatry, 162, 1483-1493.

Carlson, E. B., Putman, F. W., Ross, C. A., Torem, M., Coons, P., Dill, D., . . . Braun, B. G. (1993). Validity of the Dissociative Experience Scale in screening for multiple personality disorder: A multicenter study. American Journal of Psychiatry, 150, 1030-1036.

Cash, A. (2006). Posttraumatic stress disorder. Hoboken, NJ : Wiley.

Feuerlein, W., Ringer, C., Küfner, H. \& Antons, K. (1979). Diagnosis of alcoholism: The Munich Alcoholism Test (MALT). Currents in Alcoholism, 7, 137-147.

Frisman, L., Ford, J., Hsui-Ju, L., Mallon, S. \& Chang, R. (2008). Outcomes of trauma treatment using the TARGET model. Journal of Groups in Addiction \& Recovery, 3, 285-303.

Frodl, T. \& O'Keane, V. (2013). How does the brain deal with cumulative stress? A review with focus on developmental stress, HPA axis function and hippocampal structure in humans. Neurobiology of Disease, 52, 24-37.
Harrington, T. \& Newman, E. (2007). The psycho- metric utility of two self-report measures of PTSD among women substance users. Addictive Behaviors, 32, 2788-2798.

Harris, M. (1998). Trauma recovery and empowerment: A clinician's guide to for working with women in groups. New York, NY: Free Press.

Hase, M. (2010). CravEx: An EMDR approach to treat substance abuse and addiction. In M. Luber (Ed.), Eye movement desensitization and (EMDR) scripted protocols: Special populations (pp. 467-488). New York, NY : Springer Publishing.

Hase, M., Schallmayer, S. \& Sack, M. (2008). EMDR reprocessing of the addiction memory: Pretreatment, posttreatment, and 1-month follow-up. Journal of EMDR Practice and Research, 2(3), 170-179.

Hayes, J. P., Hayes, S. M. \& Mikedis, A. M. (2012). Quantitative meta-analysis of neural activity in posttraumatic stress disorder. Biology of Mood \& Anxiety Disorders, 2, 1-13.

Hien, D. A., Jiang, H., Campbell, A. N., Hu, M. C., Miele, G. M., Cohen, L. R., . . . Nunes, E. V. (2010). Do treatment improvements in PTSD severity affect substance use outcomes? A secondary analysis from a randomized clinical trial in NIDA's Clinical Trials Network. American Journal of Psychiatry, 167, 95-101.

Jacobsen, L. K., Southwick, S. M. \& Kosten, T. R. (2001). Substance use disorders in patients with posttraumatic stress disorder: A review of the literature. American Journal of Psychiatry, 158, 1184-1190.

Kessler, R. C, Sonnega, A., Bromet, E., Hughes, M. \& Nelson, C. B. (1995). Posttraumatic stress disorder in the National Comorbidity Survey. Archives of General Psychiatry, 52, 1048-1060.

Lecrubier, Y., Sheehan, D. V. \& Weiller, E. (1997). The Mini International Neuropsychiatric Interview (MINI). A short diagnostic structured interview: Reliability and validity according to the CIDI. European Psychiatry, 12, 224-231.

Marich, J. (2009). EMDR in the addiction continuing care process. Case study of a cross-addicted female's treatment and recovery. Journal of EMDR Practice and Research, 3(2), 98-106.

McCauley, J. L., Killeen, T., Gros, D. F., Brady, K. T. \& Back, S. E. (2012). Posttraumatic stress disorder and cooccurring substance use disorders: Advances in assessment and treatment. Clinical Psychology, 19(3), 283-304.

McDonald, S. D. \& Calhoun, P. S. (2010). The diagnostic accuracy of the PTSD Checklist: A critical review. Clinical Psychology Review, 30, 976-987.

Miller, D. \& Guidry, L. (2001). Addictions and trauma recovery: Healing the body, mind, and spirit. New York, NY : Norton.

Miller, R. (2010). The feeling-state theory of impulsecontrol disorders and the impulse-control disorder protocol. Traumatology, 16(3), 2-10.

Miller, R. (2012). Treatment of behavioral addictions utilizing the feeling-state addiction protocol: A multiple baseline study. Journal of EMDR Practice and Research. Journal of EMDR Practice and Research, 6(4), 159-169. 
Mills, K. L., Teesson, M., Back, S. E., Brady, K. T., Baker, A. L., Hopwood, S. \& Ewer, P. L. (2012). Integrated exposure-based therapy for co-occurring posttraumatic stress disorder and substance dependence: A randomized controlled trial. Journal of the American Medical Association, 308(7), 690-699.

Najavits, L. M. \& Hien, D. (2013). Helping vulnerable populations: A comprehensive review of the treatment outcome literature on substance use disorder and PTSD. Journal of Clinical Psychology, 69(5), 433-479.

Omaha, J. (1998, July). Chemotion and EMDR. Paper presented at 1998 EMDR International Association Conference, EMDR-Treatment and Transformation, Baltimore, MD.

Oren, E. \& Solomon, R. (2012). EMDR therapy: An overview of its development and mechanisms of action. European Review of Applied Psychology, 63, 197-203.

Pagani, M., Högberg, G., Fernandez, I. \& Siracusano, A. (2013). Correlates of EMDR therapy in functional and structural neuroimaging: A critical summary of recent findings. Journal of EMDR Practice and Research, 7(1), 29-38.

Perez-Dandieu, B., \& Tapia, G. (2014). Treating trauma in addiction with EMDR: A pilot study. Journal of Psychoactive Drugs, 46(4), 303-309.

Popky, A. J. (2005). DeTUR, an urge reduction protocol for addictions and dysfunctional behaviors. In R. Shapiro (Ed.), EMDR solutions: Pathways to healing (pp. 167-188). New York, NY : Norton.

Popky, A. J. (2010). The desensitization of triggers and urge processing (DeTUR) protocol. In M. Luber (Ed.), Eye movement desensitization and reprocessing (EMDR) scripted protocols: Special populations (pp. 489-511). New York, NY : Springer Publishing.

Riggs, D. S. \& Foa, E. B. (2008). Treatment for co-morbid post-traumatic stress disorder and substance use disorders. In S. H. Stewart \& P. J. Conrod (Eds.), Anxiety and substance use disorders: A vicious cycle of co-morbidity (pp. 119-137). New York, NY : Springer Publishing.

Roberts, N. P., Roberts, P. A., Jones, N. \& Bisson, J. I. (2015). Psychological interventions for post-traumatic stress disorder and comorbid substance use disorder: A systematic review and meta-analysis. Clinical Psychology Review, 38, 25-38.

Saladin, M. E., Brady, K. T., Dansky, B. S. \& Kilpatrick, D. G. (1995). Understanding comorbidity between PTSD and substance use disorders: Two preliminary investigations. Addictive Behaviors, 20(5), 643-655.

Shapiro, F. (1995). Eye movement desensitization and reprocessing: Basic principles, protocols and procedures. New York, NY : Guilford Press.
Shapiro, F. (2001). Eye movement desensitization and reprocessing: Basic principles, protocols and procedures ( $2^{\mathrm{e}}$ éd.). New York, NY : Guilford Press.

Shapiro, F. \& Maxfield, L. (2002). Eye movement desensitization and reprocessing (EMDR): Information processing in the treatment of trauma. Journal of Clinical Psychology, 58, 933-946.

Sheehan, D. V., Lecrubier, Y., Harnett-Sheehan, K., Janavs, J., Weiller, E., Bonara, L. I., . . . Dunbar, G. C. (1997). The validity of the Mini-International Neuropsychiatric Interview (MINI) according to the SCID-P and its reliability. European Psychiatry, 12, 232-241.

Solomon, R. M. \& Shapiro, F. (2008). EMDR and the adaptive information processing model. Journal of EMDR Practice and Research, 2, 315-325.

Triffleman, E. (2000). Gender differences in a controlled pilot study of psychosocial treatments in substance dependent patients with posttraumatic stress disorder: Design considerations and outcomes. Alcoholism Treatment Quarterly, 18, 113-126.

Triffleman, E., Carroll, K. \& Kellogg, S. (1999). Substance dependence posttraumatic stress disorder therapy. Journal of Substance Abuse Treatment, 17, 3-14.

Van Dam, D., Ehring, T., Vedel, E. \& Emmelkamp, P. M. (2010). Validation of the Primary Care Posttraumatic Stress Disorder screening questionnaire (PC-PTSD) in civilian substance use disorder patients. Journal of Substance Abuse Treatment, 39, 105-113.

Van Ijzendoorn, M. H., \& Schuengel, C. (1996). The measurement of dissociation in normal and clinical populations: meta-analytic validation of the Dissociative Experience Scale (DES). Clinical Psychology Review, 16(5), 365-382.

Woon, F. L., Sood, S. \& Hedges, D. W. (2010). Hippocampal volume deficits associated with exposure to psychologi- cal trauma and posttraumatic stress disorder in adults: A meta-analysis. Progress in Neuro-Psychopharmacology \& Biological Psychiatry, 34, 1181-1188.

Zweben, J., \& Yeary, J. (2006). EMDR in the treatment of addiction. Journal of Chemical Dependency Treatment, 8(2), $115-127$.

Merci d'adresser toute correspondence concernant cet article à Claire Kullack, University of Western Australia, Community, Culture and Mental Health Unit, School of Psychiatry and Clinical Neurosciences, T Block, L7, 1 Alma Street, Fremantle, WA 6160, Australie. Courriel : claire. kullack@uwa.edu.au 